\title{
Vitamin D Deficiency in Pregnancy and Its Effect on Maternal and Perinatal Outcome
}

\author{
Harpreet Kaur ${ }^{1}$, Sushmita Sharma ${ }^{2}$, Minni Verma ${ }^{3}$
}

\begin{abstract}
Background: Vitamin D deficiency is a common endocrinological problem. Maternal vitamin D insufficiency during pregnancy has been associated with many pregnancy-related complications like preeclampsia, gestational diabetes (GDM), preterm labor, and low birth weight (LBW). Maternal vitamin D deficiency can lead to poor skeletal growth and reduced bone mineral density in children.

Aims and objectives: To study the prevalence of vitamin D deficiency in antenatal women and to study an association between vitamin D deficiency and maternal and neonatal complications.

Materials and methods: A prospective study of 100 antenatal women presenting to obstetrics and gynaecology OPD at GSMCH, Banur. Out of these, data from 94 women were finally analyzed. For the rest of the women, follow-up and intrapartum and neonatal details were not available, so they were excluded from the study.

Results: The prevalence of vitamin D deficiency in the present study was $79.8 \%$. The rate of small for gestational age babies (SGA) and oligohydramnios was significantly higher in the study group. There was no significant difference in the incidence of GDM, preterm labor, and preterm premature rupture of membranes (PTPROM).

Conclusion: Antenatal vitamin D deficiency is associated with hypertensive disorders of pregnancy (HDP), SGA, oligohydramnios, and LBW. So, routine vitamin $\mathrm{D}$ supplementation should be recommended in the antenatal period.

Keywords: Hypertensive disorders of pregnancy, Low birth weight, Perinatal outcome, Pregnancy induced hypertension, Small for gestational age babies, Vitamin D deficiency.

International Journal of Infertility and Fetal Medicine (2020): 10.5005/jp-journals-10016-1197
\end{abstract}

\section{INTRODUCTION}

Vitamin D deficiency is currently a global problem. Vitamin $D$ is a fatsoluble hormone precursor that is mainly synthesized in the skin by sun exposure or can be obtained by dietary sources like egg yolk, milk, fish, and plants. $25-\mathrm{OH}$ vitamin $\mathrm{D}$ is the major storage form of vitamin $\mathrm{D}$ in the body. Recently, it has been shown that vitamin D deficiency is common during pregnancy, especially among the high-risk groups including vegetarians, women with limited sun exposure, and certain ethnic groups. Ethnic groups at risk of vitamin D deficiency include those from the Indian subcontinent and the Middle East. Vitamin D deficiency in pregnancy is associated with many pregnancy-related complications like preeclampsia, gestational diabetes (GDM), preterm labor, and low birth weight (LBW). Also, neonatal vitamin D levels are largely dependent on maternal vitamin D status. So, infants of mothers with vitamin $\mathrm{D}$ deficiency are also at risk of vitamin $\mathrm{D}$ deficiency. Maternal vitamin D deficiency can lead to disordered skeletal homeostasis, congenital rickets, and fractures in the newborn. ${ }^{1-3}$

Maternal vitamin D insufficiency is common during pregnancy and is associated with reduced bone mineral accrual in the offspring during childhood; this association is mediated partly through the concentration of umbilical venous calcium. Vitamin D supplementation of pregnant women, especially during winter months, could lead to long-lasting reductions in the risk of osteoporotic fracture in their offspring. ${ }^{4}$

Recent systematic review and meta-analysis revealed that vitamin D insufficiency is associated with an increased risk of GDM, preeclampsia, and small for gestational age infants. Pregnant women with low $25-\mathrm{OH}$ vitamin D levels had an increased risk of
1,2Department of Obstetrics and Gynaecology, Gian Sagar Medical College and Hospital, Chandigarh, India

${ }^{3}$ Department of Biochemistry, Gian Sagar Medical College and Hospital, Chandigarh, India

Corresponding Author: Harpreet Kaur, Department of Obstetrics and Gynaecology, Gian Sagar Medical College and Hospital, Chandigarh, India, Phone: +91 9872221818, e-mail: drharpreet_sidhu@hotmail. com

How to cite this article: Kaur $H$, Sharma S, Verma M. Vitamin D Deficiency in Pregnancy and Its Effect on Maternal and Perinatal Outcome. Int J Infertil Fetal Med 2020;11(1):11-15.

Source of support: Nil

Conflict of interest: None

bacterial vaginosis and lower birth weight infants. ${ }^{5}$ In a study by Sohail and Rashid, $98 \%$ of primigravidae were shown to be vitamin $\mathrm{D}$ deficient, and mean vitamin D levels in antenatal women were $10.30 \mathrm{ng} / \mathrm{mL}$ despite routine vitamin $\mathrm{D}$ prophylaxis of $400 \mathrm{IU} /$ day. So, the authors supported the routine screening of all pregnant women for vitamin D levels. ${ }^{6}$

Though biological evidence regarding a role for vitamin $D$ in reproductive outcomes is strong, and rates of vitamin D deficiency may be high among pregnant women. However, no consensus exists regarding optimum vitamin D levels in pregnancy or standard measurement of vitamin D deficiency. Currently, the American College of Obstetrics and Gynecology (ACOG) and the Institute of Medicine recommends 600IU of daily vitamin D supplementation during pregnancy to support maternal and fetal bone metabolism. ${ }^{3}$

() The Author(s). 2020 Open Access This article is distributed under the terms of the Creative Commons Attribution 4.0 International License (https://creativecommons. org/licenses/by-nc/4.0/), which permits unrestricted use, distribution, and non-commercial reproduction in any medium, provided you give appropriate credit to the original author(s) and the source, provide a link to the Creative Commons license, and indicate if changes were made. The Creative Commons Public Domain Dedication waiver (http://creativecommons.org/publicdomain/zero/1.0/) applies to the data made available in this article, unless otherwise stated. 
In a Cochrane review 2012, it was concluded that vitamin D supplementation in a single or continued dose during pregnancy increases serum vitamin $\mathrm{D}$ concentrations as measured by $25-\mathrm{OH}$ vitamin $\mathrm{D}$ at term but the clinical significance of this finding and the potential use of this intervention as a part of routine antenatal care are yet to be determined. ${ }^{7}$

\section{Aims and Objectives}

- To study the prevalence of vitamin D deficiency in antenatal women.

- To study an association between vitamin D deficiency and maternal and neonatal complications.

\section{Study Design}

A prospective study of 100 antenatal women presenting to obstetrics and gynaecology OPD at GSMCH, Banur.

\section{Materials and Methods}

The study was conducted on all antenatal cases presenting to obstetrics and gynaecology OPD at GSMCH, Banur, Patiala from June 2013 to May 2015 who consented to participate in the study. $25-\mathrm{OH}$ vitamin $\mathrm{D}_{3}$ levels were checked in 120 women at the first visit to antenatal OPD by fully automated chemiluminescence assay (Liaison Diasorin). The value of $20 \mathrm{ng} / \mathrm{mL}$ of $25-\mathrm{OH}$ vitamin $\mathrm{D}_{3}$ was taken as normal. Out of these, data from 94 women were finally analyzed. For the rest of the women, follow-up and intrapartum and neonatal details were not available, so they were excluded from the study. Women found to be vitamin $D$ deficient were prescribed a mega-dose of vitamin D as sachets of $60,000 \mathrm{IU}$ of vitamin D weekly (Rockbon vitanules, Abbott Pharmaceuticals) in addition to calcium supplementation of $1,000 \mathrm{mg}$ daily after 16 weeks of pregnancy. Maternal antenatal complications and any significant intrapartum complications were studied in all cases and comparison was made between those with normal levels of vitamin D and the ones with low vitamin D levels. A record of perinatal outcome was made in all cases.

Outcome studied were:

- Prevalence of vitamin D deficiency in antenatal women.

- Relation of vitamin $D$ deficiency with hypertensive disorders of pregnancy (HDP), GDM, small for gestational age babies (SGA), preterm delivery, preterm premature rupture of membranes (PTPROM), stillbirths, LBW, and any immediate neonatal complications.

Hypertensive disorders of pregnancy was defined as blood pressure $\geq 140 / 90 \mathrm{~mm} \mathrm{Hg}$, measured on two occasions 6 hours apart, associated with proteinuria or other signs of preeclampsia (as defined in NICE guidelines 2010). Gestational diabetes was defined deranged glucose tolerance test after $75 \mathrm{~g}$ glucose load detected after 20 weeks of pregnancy (as per the NICE guideline 2015). Small for gestational age babies was labeled when estimated fetal weight was below the 10th percentile of the expected fetal weight at given gestation based on ultrasonography (USG) findings (as per the Green Top guideline No. 13). Preterm labor was defined as the spontaneous onset of labor between $28+0$ and $33+6$ weeks, and PTPROM as preterm rupture of membranes before onset of labor and occurring before 34 completed weeks. Babies born dead after 28 weeks period of gestation (POG) were defined as stillbirths and LBW was defined as birth weight below 2 SD of the expected birth weight.

\section{Exclusion Criteria}

- Multiple pregnancies.

- Women with chronic renal disease.

- Women with liver disease.

- Women on antiepileptics and antituberculosis therapy.

- Women with albinism.

- Women with known endocrinopathy affecting vitamin D or calcium metabolism like parathormone deficiency.

\section{Statistical Methods}

Descriptive statistical analysis has been carried out in the present study. Results on continuous measurements are presented as mean \pm SD (min-max) and results on categorical measurements are presented as number (\%). Significance is assessed at the $5 \%$ level of significance. Student's $t$-test (two-tailed, independent) has been used to determine the significance of the study parameters on a continuous scale between the two groups (intergroup analysis) on metric parameters and Chi-square/Fisher's exact test has been used to find the significance of the study parameters on a categorical scale between two or more groups. $p$ value of $<0.05$ was taken as significant.

\section{Results}

The prevalence of vitamin D deficiency in the present study was 79.8\% (Table 1).

The rate of SGA and oligohydramnios was significantly higher in the study group. There was no significant difference in the incidence of GDM, preterm labor, and PTPROM (Tables 2 and 3).

Table 4 shows the correlation of vitamin D levels with and antenatal complications. The mean birth weight was 2.782 (2.6962.868). In all the groups, mean birth weight was low in women with very low vitamin $\mathrm{D}$ levels.

\section{Discussion}

Vitamin $D$ is important for bone metabolism in addition to calcium and other factors. As fetal and neonatal vitamin D levels are dependent on maternal vitamin D status, recently there is a lot of research regarding the correlation of vitamin D levels with pregnancy outcome. Various studies have shown an increased risk of pregnancy complications like pregnancy induced hypertension $(\mathrm{PIH})$, intrauterine growth restriction (IUGR), GDM, and preterm labor in women who are having low serum vitamin D levels. ${ }^{3,4}$

Table 1: Demographic profile of women

\begin{tabular}{lll}
\hline \multicolumn{5}{c}{ Vit D deficient, $n=75(\%)$} & Normal vit D levels, $n=19(\%)$ \\
\hline Age & & $12(63.2)$ \\
$20-25$ & $43(57.3)$ & $6(31.6)$ \\
$26-30$ & $21(28.1)$ & $01(5.2)$ \\
$31-35$ & $11(14.6)$ & \\
Parity & & $11(57.9)$ \\
Nullipara & $51(68)$ & $8(42.1)$ \\
$1-2$ & $24(32)$ & $6(31.6)$ \\
Body mass index & $7(36.8)$ \\
$18-24$ & $26(34.6)$ & $4(21)$ \\
$25-30$ & $28(37.4)$ & $2(10.6)$ \\
$30-40$ & $18(24.0)$ & \\
40 & $3(4.0)$ &
\end{tabular}


Vitamin D Deficiency in Pregnancy

Table 2: Antenatal complications

\begin{tabular}{|c|c|c|c|c|}
\hline & Vitamin D deficient, ${ }^{*} n=75$ (\%) & Normal Vit D levels, ${ }^{*} n=19(\%)$ & 95\% confidence interval & $p$ value \\
\hline HDP & $14(18.67)$ & $01(5.2)$ & -0.2482 to 0.1681 & 0.6888 \\
\hline GDM & $04(5.4)$ & $01(5.2)$ & -0.2268 to 0.1900 & 0.8541 \\
\hline SGA & $23(30.67)$ & $01(5.2)$ & -0.5862 to -0.2437 & $<0.000^{* * * *}$ \\
\hline Oligohydramnios & $20(26.7)$ & $03(15.8)$ & -0.3971 to 0.002932 & $0.0467^{*}$ \\
\hline Preterm labor & $09(12)$ & $03(15.8)$ & -0.3738 to 0.03032 & 0.0838 \\
\hline PTPROM & $12(16)$ & $02(10.6)$ & -0.3529 to 0.05441 & 0.1336 \\
\hline $\mathrm{APH}$ & 01 (Abruptio) (1.4) & 0 & 0.2249 to 0.1920 & 0.8693 \\
\hline PPH & 02 (1 atonic, 1 retained placenta) (2.7) & 0 & - & - \\
\hline
\end{tabular}

*Total value may not match as many women had more than one complication

The rate of SGA and oligohydramnios was significantly higher in the study group. There was no significant difference in the incidence of GDM, preterm labor, and PTPROM

Table 3: Mode of delivery

\begin{tabular}{lll}
\hline & $\begin{array}{l}\text { Vitamin D deficient } \\
(n=75)\end{array}$ & $\begin{array}{l}\text { Normal vitamin D } \\
(n=19)\end{array}$ \\
\hline Term vaginal delivery & $35(46.7)$ & $11(57.9)$ \\
PTVD & $14(18.7)$ & $03(15.8)$ \\
LSCS & $26(34.7)$ & $05(26.3)$ \\
Fetal distress & 9 & 1 \\
Non-progress of labor & 3 & 1 \\
APH & 1 & 0 \\
Others & 13 & 03 \\
\hline
\end{tabular}

Currently, there are no data to support routine screening for vitamin D deficiency in pregnancy in terms of health benefits or cost-effectiveness. Keeping the cost of the test in mind, offering the test to at-risk women may be cost-effective compared with offering universal supplementation, particularly as treatment is regarded as being very safe. At present, there are no data to support a strategy of measurement followed by treatment in the general female population. ${ }^{8,9}$

The prevalence of vitamin D deficiency in the present study was $79.8 \%$. Various studies have shown the percentage of vitamin D deficiency in pregnant females varying from 21 to $100 \% .^{10-12}$ In a study by Marwaha et al., ${ }^{13} 96 \%$ of urban Indian women were vitamin $D$ deficient.

The present study showed an increased incidence of HDP in women who had deficient vitamin D levels (18.6 vs $5.2 \%$ ) but the difference was not significant. There is conflicting evidence about whether hypovitaminosis $D$ in pregnancy is associated with hypertension and preeclampsia. In a systematic review and meta-analysis of nine studies, Fariba et al. ${ }^{5}$ had shown a significant association between vitamin D deficiency and preeclampsia (pooled OR of 1.79). The mechanism implicated in the causative association of vitamin $D$ deficiency and preeclampsia is related to its immuno-modulatory effects. This is supported by the observation of decreased incidence of preeclampsia in women given vitamin D supplementation and incidence of PIH was found to be higher during winter and rainy seasons when vitamin $D$ deficiency is also higher. $^{14-17}$ In Cochrane review 2012, there was one trial of 400 women reporting an association with preeclampsia which showed no difference in the incidence of preeclampsia in study and control groups. ${ }^{7}$ When all women with low vitamin D levels were analyzed separately, there was no correlation between vitamin D levels and the incidence of HDP. A maximum number of patients of HDP were seen in the group with vitamin D levels in the range of 5-15 ng/ $\mathrm{mL}$. In a study by Bener et al., maximum patients with preeclampsia were seen in the moderate deficiency group. ${ }^{18}$ So, it is difficult to say whether vitamin $D$ is an independent risk factor for preeclampsia. A prospective cohort study failed to show an association. ${ }^{16}$ Lisa et al. have shown a dose-response relationship between vitamin $\mathrm{D}$ levels at $<22$ weeks of gestation and the risk of development of preeclampsia. ${ }^{19}$ However, two meta-analyzes, including a metaanalysis of 31 studies, demonstrated that vitamin D insufficiency was associated with preeclampsia and SGA infants., 20

As per WHO guidelines on vitamin D supplementation during pregnancy, ${ }^{21}$ vitamin $\mathrm{D}$ supplementation is not recommended during pregnancy to prevent the development of preeclampsia and its complications (strong recommendation).

There was an increased prevalence of SGA and oligohydramnios in the vitamin D deficient group (30 vs $5 \%$ and 26 vs 16\%). The majority (87\%) of the cases of SGA were seen in the group with moderate vitamin D deficiency. Maternal vitamin D levels have been shown to positively correlate with birth weight centiles. ${ }^{22}$ The largest cohort study (3,730 Amsterdam women) found the odds of birthing a baby small for gestational age was higher among women with severe vitamin $D$ deficiency in early pregnancy $(<12 \mathrm{ng} / \mathrm{L})$. This large cohort study in the Netherlands ${ }^{23}$ found that women with severe vitamin $D$ deficiency $(<30 \mathrm{nmol} / \mathrm{L})$ bore infants who weighed $114 \mathrm{~g}$ less on average than women with adequate vitamin $\mathrm{D}(>50$ $\mathrm{nmol} / \mathrm{L}$ ) and had 2.4 times the odds of SGA. Another study found that maternal vitamin $D$ levels of $<37.5 \mathrm{nmol} / \mathrm{L}$ in the first half of pregnancy were associated with an adjusted odds ratio of 7.5 for SGA infants in white women, but not in black women. ${ }^{24}$

However, other studies demonstrated no relationship between maternal vitamin D levels in the first trimester and birthweight but did demonstrate that low vitamin D levels in late pregnancy were associated with reduced intrauterine long bone growth and lower gestational age at delivery. ${ }^{25}$ Low vitamin D levels during pregnancy are associated with reduced bone mass in childhood. ${ }^{4}$

A meta-analysis published in BMJ, using the most adjusted odds ratio, showed a significant association between small for gestational age infants and $25-\mathrm{OH}$ vitamin $\mathrm{D}$ insufficiency compared with the comparison group (OR 1.85, 95\% Cl 1.52-2.26). ${ }^{5}$

There was an increased incidence of preterm birth and PTPROM in the group with vitamin D deficiency, though the difference was not significant. Literature shows conflicting results regarding the association between vitamin $D$ deficiency and the risk of preterm birth. Due to the association with increased markers of inflammation and vitamin D deficiency, some studies have shown an increased 


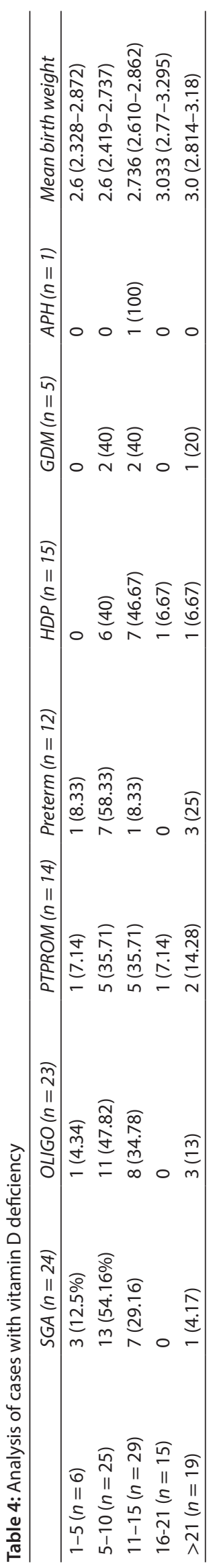

incidence of preterm labor in women with vitamin D deficiency. A Japanese retrospective study found a lower mean $25(\mathrm{OH}) \mathrm{D}$ among women in the third trimester that had been hospitalized for preterm labor earlier in the pregnancy but delivered at term compared with those who had not been. ${ }^{26}$ Singh et al. studied the relationship between vitamin D deficiency and preterm labor in the Indian population. ${ }^{27}$ Among 100 pregnant women studied, the risk of preterm was higher in the hypovitaminosis group (12 vs $30 \%, p=$ 0.005). Vitamin D deficiency is associated with an increased risk of both spontaneous and medically induced preterm birth. This may be linked to its ability to reduce bacterial infections by inducing the production of the antibacterial compound, cathelicidin, in placental cells. Data from the epidemiology of vitamin D study (EVITA) have shown that the confounder-adjusted risk of preterm birth was highest when serum 25-hydroxyvitamin D was $<20 \mathrm{ng} /$ $\mathrm{mL}$, declined as 25-hydroxy vitamin D increased to approximately 36 $\mathrm{ng} / \mathrm{mL}$, and then plateaued. Findings were similar for spontaneous or medically indicated preterm birth and preterm birth at $<34$ weeks of gestation. ${ }^{28}$

Various studies have shown a variable association between vitamin D levels and the risk of GDM. ${ }^{5,9,18}$ In the present study, there was no significant association between vitamin D deficiency and the risk of GDM.

\section{Conclusion}

Vitamin D deficiency is a common endocrinopathy. Antenatal vitamin D deficiency is associated with HDP, IUGR, GDM, preterm labor, PTPROM, and low childhood bone density. The present study showed a high prevalence of vitamin D deficiency (79.8\%) in the antenatal women and there was a statistically significant higher rate of SGA babies in women showing vitamin D deficiency. So, routine vitamin D supplementation, as recommended by ACOG, can help to prevent many pregnancy-associated complications to some extent.

\section{References}

1. Pawley N, Bishop NJ. Prenatal \& infant predictors of bone health: the influence of vitamin D. Am J Clin Nutr 2004;80(6):1748S-1751SS. DOI: 10.1093/ajcn/80.6.1748S.

2. Gale CR, Robinson SM, Harvey NC, et al. Maternal vitamin D status during pregnancy and child outcomes. Princess Anne hospital study group. Eur J Clin Nutr 2008;62(1):68-77. DOI: 10.1038/sj.ejcn.1602680.

3. Vitamin $D$ screening and supplementation during pregnancy. Committee opinion no. 495. American college of obstetricians \& gynaecologists. Obstet Gynaecol 2011;118(1):197-198. DOI: 10.1097/ AOG.0b013e318227f06b.

4. Javaid MK, Crozier SR, Harvey NC, et al. Maternal vitamin D status during pregnancy and childhood bone mass at age 9 years: a longitudinal study. Lancet 2006;367(9504):36-43. DOI: 10.1016/ S0140-6736(06)67922-1.

5. Aghajafari F, Nagulesapillai T, Ronksley PE, et al. Association between maternal serum 25-hydroxyvitamin D level and pregnancy and neonatal outcomes: Systematic review and meta-analysis of observational studies. BMJ 2013;346(mar26 4):10. DOI: 10.1136/bmj. f1169.

6. Sohail S, Rashid S. Serum $25-\mathrm{OH}$ vitamin D level is the best predictor of vitamin D deficiency during pregnancy. Professional Med J 2013;20(1):78-81.

7. De-Regil LM, Palacios C, Ansary A, et al. Vitamin D supplementation for women during pregnancy. Cochrane Database Syst Rev 2012(2):CD008873.

8. Yu CKH, Sykes L, Sethi M, et al. Vitamin D deficiency and supplementation during pregnancy. Clin Endocrinol (Oxf) 2009;70(5):685-690. DOI: 10.1111/j.1365-2265.2008.03403.x. 
9. Urrutia RP, Thorp JM. Vitamin D in pregnancy: current concepts. Curr Opin Obstet Gynecol 2012;24(2):57-64. DOI: 10.1097/ GCO.0b013e3283505ab3.

10. Looker $A C$, Johnson $C L$, Lacher $D A$, et al. Vitamin $D$ status: United States, 2001-2006. NCHS Data Brief 2011(59):1-8.

11. Ginde AA, Sullivan AF, Mansbach JM, et al. Vitamin D insufficiency in pregnant and nonpregnant women of childbearing age in the United States. Am J Obstet Gynecol 2010;202(5):436.e1-8. DOI: 10.1016/j. ajog.2009.11.036.

12. Hamilton SA, McNeil R, Hollis BW, et al. Profound vitamin D deficiency in a diverse group of women during pregnancy living in a sun-rich environment at latitude 32 degrees N. Int J Endocrinol 2010;2010:917428. DOI: 10.1155/2010/917428.

13. Marwaha RK, Tandon N, Chopra S, et al. Vitamin D status in pregnant Indian women across trimesters and different seasons and its correlation with neonatal serum 25-hydroxyvitamin $\mathrm{D}$ levels. $\mathrm{Br} \mathrm{J}$ Nutr 2011(9):1-7. DOI: 10.1017/S000711451100170X.

14. Haugen M, Brantsæter AL, Trogstad L, et al. Vitamin D supplementation and reduced risk of preeclampsia in nulliparous women. Epidemiology 2009;20(5):720-726. DOI: 10.1097/EDE.0b013e3181a70f08.

15. TePoel MR, Saftlas AF, Wallis AB. Association of seasonality with hypertension in pregnancy: asystematic review. J Reprod Immunol 2011;89(2):140-152. DOI: 10.1016/j.jri.2011.01.020.

16. Shand A, Nassar N, Von Dadelszen P, et al. Maternal vitamin D status in pregnancy and adverse pregnancy outcomes in a group at high risk for pre-eclampsia. BJOG 2010;117(13):1593-1598. DOI: 10.1111/j.14710528.2010.02742.x.

17. Baker AM, Haeri S, Camargo CA, et al. A nested case-control study of midgestation vitamin $D$ deficiency and risk of severe preeclampsia.J Clin Endocrinol Metab 2010;95(11):5105-5109. DOI: 10.1210/jc.20100996.

18. Bener A, Al-Hamaq AO, Saleh NM. Association between vitamin D deficiency and adverse pregnancy outcome-global comparisons. Int J Women's Health 2013;5:523-531. DOI: 10.2147/IJWH.S51403.
19. Bodnar LM, Catov JM, Simhan HN, et al. Maternal vitamin D deficiency increases the risk of preeclampsia. J Clin Endocrinol Metabol 92(9):3517-3522. DOI: 10.1210/jc.2007-0718.

20. Wei SQ, Qi HP, Luo ZC, et al. Maternal vitamin D status and adverse pregnancy outcomes: a systematic review and metaanalysis. J Matern Fetal Neonatal Med 2013;26(9):889-899. DOI: 10.3109/14767058.2013.765849.

21. WHO. Guideline: Vitamin D supplementation in pregnant women. Geneva: World Health Organization; 2012.

22. Robinson $\mathrm{CJ}$, Wagner $\mathrm{CL}$, Hollis BW, et al. Maternal vitamin $\mathrm{D}$ and fetal growth in early-onset severe preeclampsia. Am J Obstet Gynecol 2011;204(6):556. DOI: 10.1016/j.ajog.2011.03.022.

23. Leffelaar ER, Vrijkotte TG, van Eijsden M. Maternal early pregnancy vitamin $D$ status in relation to fetal and neonatal growth: results of the multi-ethnic Amsterdam born children and their development cohort. Br J Nutr 2010;104(1):108-117. DOI: 10.1017/S000711451000022X.

24. Bowyer L, Catling-Paull C, Diamond T, et al. Vitamin D, PTH and calcium levels in pregnant women and their neonates. Clin Endocrinol (Oxf) 2009;70(3):372-377. DOI: 10.1111/j.1365-2265.2008.03316.x.

25. Morley R, Carlin JB, Pasco JA, et al. Maternal 25-hydroxyvitamin D and parathyroid hormone concentrations and offspring birth size. J Clin Endocrinol Metab 2006;91(3):906-912. DOI: 10.1210/jc.20051479.

26. Shibata M, Suzuki A, Sekiya T, et al. High prevalence of hypovitaminosis D in pregnant Japanese women with threatened premature delivery. J Bone Miner Metab 2011;29(5):615-620. DOI: 10.1007/s00774-0110264-x.

27. Singh J, Hariharan C, Bhaumik D. Role of vitamin D in reducing the risk of preterm labour. Int J Reprod Contracept Obstet Gynecol 2015;4(1):86-93. DOI: 10.5455/2320-1770.ijrcog20150217.

28. Bodnar LM, Platt RW, Simhan HN, et al. Early-pregnancy vitamin $D$ deficiency and risk of preterm birth subtypes. Obstet Gynecol 2015;125(2):439-447. DOI: 10.1097/AOG.0000000000000621. 